\title{
Health screening examinations in cardiovascular risk estimation
}

\author{
KóróDI Gyula MD ${ }^{1}$
}

\begin{abstract}
The most important elements in prevention of cardiovascular (and cerebrovascular) dis-eases are screening and managing the risk factors, such as high blood pressure, high total cholesterol or high blood glucose etc.. The best way to find out the personal specific risk profile is through screening tests during annual doctor visits. Regular cardiovascu- lar and cerebrovascular screenings are very important because they help detect the risk factors in their earliest and asymptomatic stages. This way, the patients can treat their risk factors with lifestyle changes and pharmacotherapies, if appropriate, before it ulti-mately leads to the development of cardiovascular disease. For many patients, screening results can serve as a wake-up call. When the regular test comes back and the patient sees and understands the abnormal parameters, it becomes extremely personal. The idea of making lifestyle changes is not just a recommendation in a pamphlet; it is something that can impact the patient's whole life, habits and health. The best way for optimal health benefits is through regular cardiovascular screening tests beginning at age 20. Because of very high cardiovascular morbidity and mortality, early and regular screen-ings could be a basic element of preventive health care.
\end{abstract}

Keywords: cardiovascular disorders, screening examination, preventive care, risk estimation

\section{Screening examinations in general medicine}

We can define health screening as the preclinical identification of disease by means of tests or ex- aminations that can be applied rapidly. In practical medicine screening is a preventive strategy used in a population to detect a disease before the signs or symtoms of it. The intention of screening is to identify risk factors in a community, for earlier intervention and management in the hope to reduc- ing morbidity and mortality. Screening refers to a test or exam done to find a pathological condition before symptoms begin, when they are much easier to treat. The screened condition itself must be important, recognisable and clearly understood. Screening is intended for all people, in an identified target population, who do not have symptoms of the disease or the condition being screened for. Not everyone will take part in a screening program, there are factors that differ between those willing to get tested and those who are not. One hopes people with a higher risk of a disease are more likely to get screened. Which tests you need depends on your age, your sex, your family medical history and whether you have risk factors for certain diseases. For example, being overweight may increase your risk of developing diabetes or hypertension and can increase the risk of cardiovascular disease or stroke. Nowadays in Hungary's financial and economic circumstances employees can be the main target population of screening programs, because they are the key elements in GDP production.

The screening test itself must be a good one. It must be sensitive, that is to say people with the

1 Quatos Ltd. Email: korodigy@ freemail.hu

disease will correctly test positive, and it must also be specific, so that those without the disease will correctly test negative. Ideally the test would pick out all diseased individuals and be positive for that disease only. They must be also be cheap, easy, reliable and as painless as possible. For these reasons a test used in a screening program, especially for a disease with low incidence, must be very specific in addition to acceptably sensitive.

Screening has advantages and disadvantages: the decision whether to screen must be decided by balancing all factors. Screening can detect medical conditions at an early stage before symp- toms present themselves, while treatment is more effective for later detection. In the best of cases an early diagnosis can be life-saving because of the highly effective treatment modalities. Like any medical test, the tests used in screening are not perfect. The test result may incorrectly show a pos- itive for those without the disease (false positive), or negative for people who have the condition (fălse negative). Screening involves cost and use of medical resources on a majority of people who do not need treatment. False positive results and unnecessary investigations can provoke adverse effects of screening procedure: stress, anxiety, discomfort, radiation or chemical exposure. Screen- ing may identify abnormalities that would never cause a problem in a person's lifetime.

Several types of screening exist in medicine; the annual and general medical examination is a common form of preventive health care. universal check ups involve screening of all patients, and case finding involves screening a smaller group of people. Primary care is the term for the health care services that play a role in the local community. It refers to the work of general practitioners, pharmacists, and nurse practitioners. Continuity, preventive care and health education are the key characteristics of primary care, as patients usually prefer to consult the same practitioner for rou- tine check-ups. Secondary care is the health service provided by medical specialists and other pro- fessionals who generally do not have first contact with patients. Allied health professionals, such as occupational therapists also generally work in secondary care. Tertiary care 
(advanced medical investigation and treatment) is specialized consultative health care, usually for inpatients and on referral from a primary to secondary professionals.

\section{Screening examinations in cardiovascular prevention}

A family's medical history can reveal the health risk in the family and allow you to identify pat- terns that might be relevant to the patient's own health. We can use the medical history data to assess the risk of diseases, recommend changes in lifestyle habits to reduce the risk of disease and determine the frequency and type of screening exams. All the employee and the patient need to do is to answer the questionnaire about the family \& personal medical history, about the risk factors at the workplace and about the patient's lifestyle. After this it is a medical task to plan and execute the personalised health check up. In the civilised world more than half the cases of illness and the leading cause of death are cardiovascular diseases. Because of this one of the most important parts of the medical screening system is cardiovascular risk profile estimation. Numerous risk factors of cardiovascular diseases have been known, most of them relate to life style (cigarette smoking, drug abuse, excessive alcohol consumption, bad eating habits, inadequate physical activity etc.) but other environmental risk factors, genetics or geographical differences in terms of access to a health care system, also could significantly influence mortality.

using the simple European SCOrE help we can calculate the statistical risk for fatal heart attack for a period over 10 years. If we know the patient's age (aged 40 and over) sex, smoking status, blood pressure and serum cholesterol level we can estimate the 10-year risk of fatal cardio- vascular attack in percentage.

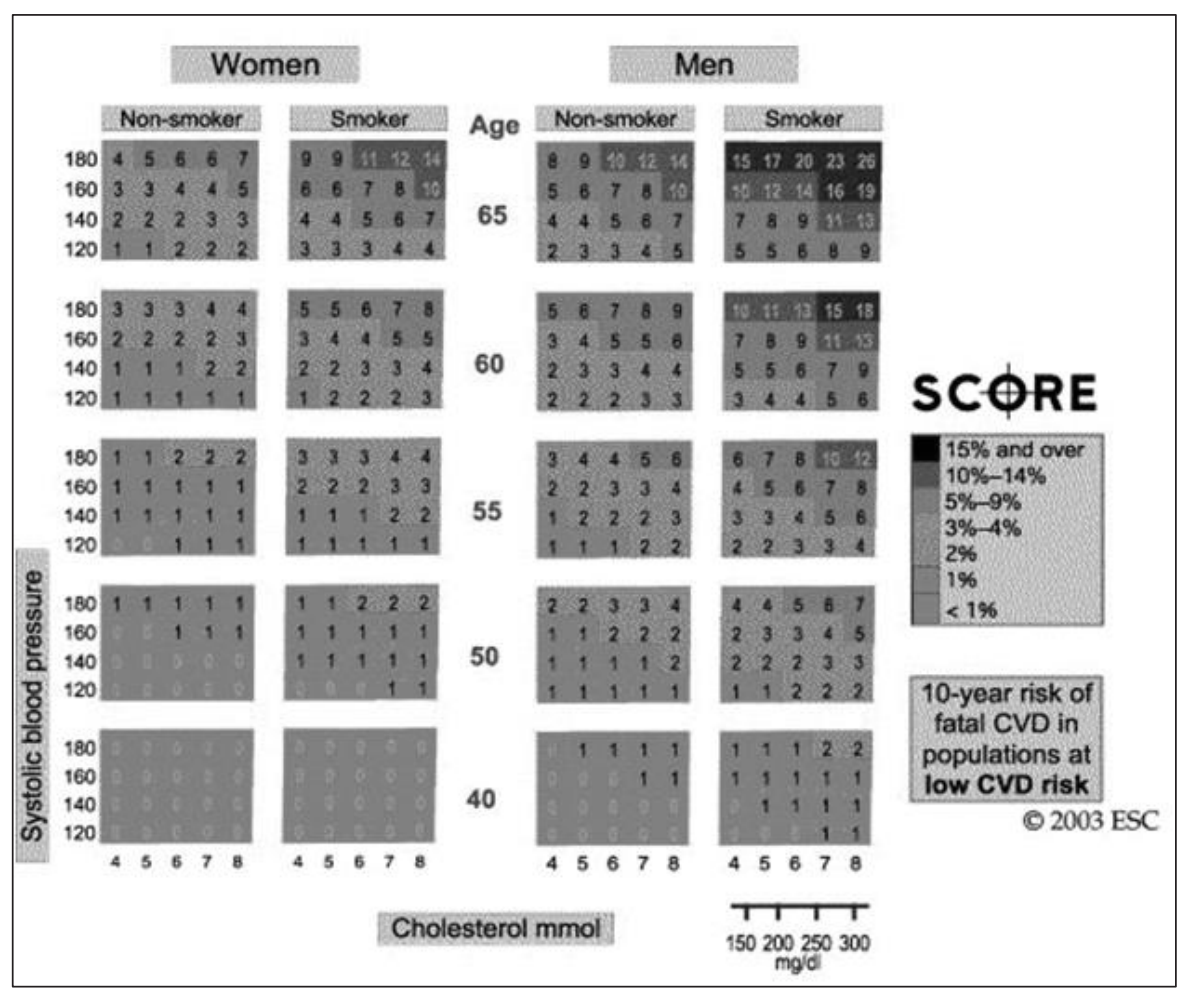

Figure 1. Systematic Coronary Risk Evaluation (SCORE), cardiovascular Risk Charts based on gender, age, total cholesterol, systolic blood pressure and smoking status

using the American Framingham (Massachusetts, uSA) study we can create a well circum- scribed prognosis of a nonfatal cardiovascular attack in the population or single persons. The risk assessment tool below uses information from the Framingham Hearth Study to predict the person's chance of having a non fatal hearth attack in the next 10 years. This tool is designed for adults aged

40

20 and older who do not have heart disease or diabetes. Since 1948(!) the subjects have continued to return to the study every year for physical examination and blood tests (nowadays ultrasound of the heart \& arteries, bone densitometry, heart \& brain CT or MrI). Since the 1990s the studies have documented a strong correlation between cardiovascular diseases and osteoporosis in special laboratory tests (homocystein, CrP, uric acid etc.)

Using these kinds of early and sensitive markers we can calculate the personal risk pofile: A small cardiovascular risk means under $15 \%$ Framingham and under 4 SCOrE percent, and we talk of high risk above $20 \%$ Framingham and above 5 SCOrE percent.

In October 2011 a new model was prepared from fresh data and was launched at the 2011

EACTS meeting in Lisbon. The model is called EuroSCOrE II - this online calculator has been updated to use this new model. It calculates the renal impairment, the extracardial angiopathy, the poor mobility, previous cardiac surgery, chronic lung disease, endocarditis, critical preoperative status, diabetes on insulin, cardiac and operation related factors. using the 


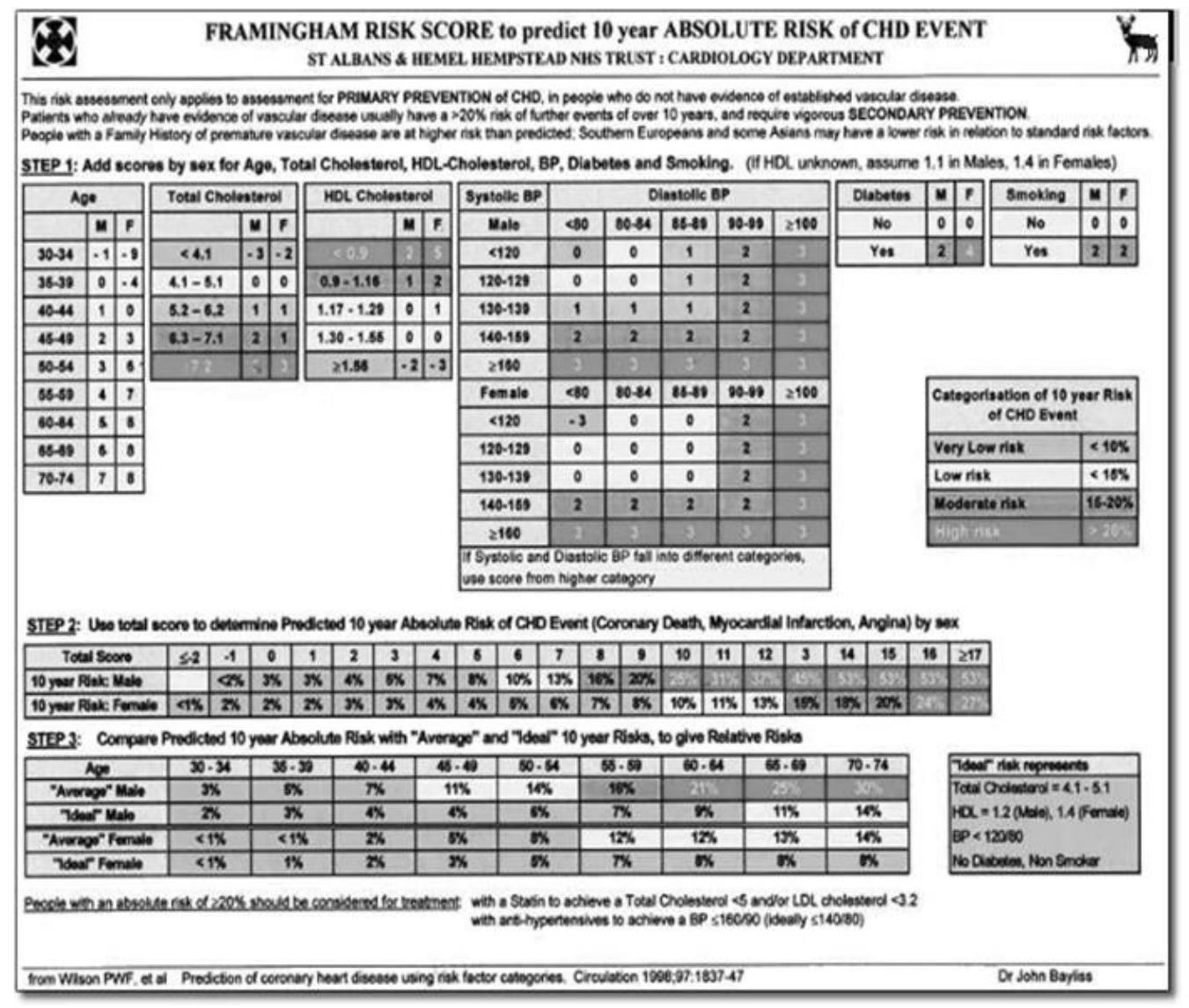

Figure 2. The Framingham cardiovascular risk core

\section{Summary}

The cross disciplinary nature of cardiovascular disease needs strong cooperation between a lot of medical specialties in prevention, treatment and rehabilitation. This kind of cooperation must create interdisciplinary teams of academic staff from the basic sciences, together with clinicians in cardiology and stroke therapy also visiting experts in the field of cardiovascular prevention and rehabilitation. The delivery of modern health care depends on groups of professionals and parapro- fessionals coming together as cardiovascular teams, who systematically provide personal and pop- ulation-based preventive care and service to the entire society. Preventive care refers to measures taken to prevent cardiovascular and other diseases, rather than treating their symptoms. The term cardiovascular preventive care contrasts in method from curative medicine, and works at the level of population health rather than individual health. Preventive medicine deals with a healthy popu- lation where the costs need even more careful examination and application. For an intervention to be applied widely it generally needs to be affordable and highly cost effective.

This paper was supported by the János Bolyai Research Scholarship of the Hungarian Academy of Sciences.

\section{References}

ANDErSON, K. M. et al. (1991): An Updated Coronary Risk Profile. A Statement for Health Profes- sionals. In: Circulation. 1991;83:356-362. [PubMed] https://doi.org/10.1161/01.CIR.83.1.356 41

CONrOy, r. M. et al. (2003): Estimation of ten-year risk of fatal cardiovascular disease in Europe: the SCOrE project. In: Eur Heart J. 2003;24:987-1003. [PubMed] https://doi.org/10.1016/S0195-668X(03)00114-3

FErDINAND, K. C. (2005): Managing cardiovascular risk in minority patients. In: J National Med Assoc. 2005;97:459-466.

FLETCHEr, G. F. et al. (1996): Statement on exercise: benefits and recommendations for physical activity programs for all Americans: a statement for health professionals by the Committee on Ex- ercise and Cardiac rehabilitation of the Council on Clinical Cardiology, American Heart Associa- tion. Circulation. 1996;94:857862. [PubMed] https://doi.org/10.1161/01.CIR.94.4.857

Népegészségügyi Jelentés 2003., Johan Béla Országos Epidemiológiai Központ, Budapest.

rOQuES, F. et al. (1999): risk factors and outcome in European cardiac surgery: analysis of the Euro- SCOrE multinational database of 19030 patients. Eur J Cardiothorac Surg. 1999 Jun;15(6):816-22; discussion 822-3.

rOQuES, F. et al. (2003): The logistic EuroSCOrE. In: Eur Heart J. 2003 May;24(9):882-3.https://doi.org/10.1016/

S0195-668X(02)00799-6 